\title{
Chinese solar physics gliding into the space age
}

\author{
PengFei Chen ${ }^{1,2^{*}}$ \\ ${ }^{1}$ School of Astronomy \& Space Science, Nanjing University, Nanjing 210023, China; \\ ${ }^{2}$ Key Laboratory of Modern Astronomy \& Astrophysics (Nanjing University), Ministry of Education, Nanjing 210023, China
}

Received July 19, 2018; accepted July 26, 2018; published online August 10, 2018

Citation: P. F. Chen, Chinese solar physics gliding into the space age, Sci. China-Phys. Mech. Astron. 61, 109631 (2018), https://doi.org/10.1007/s11433-0189282-y

On May 4, 2018, as the chief engineer, Prof. SUN Weigang, signed his name on the Review Report, the Solar $\mathrm{H} \alpha$ Imaging Spectrometer spacecraft was finally approved by the Chinese Space Agency. This is the second spacecraft designated for solar observations in China, coming on the heels of the Advanced Space-born Solar Observatory (ASO-S), which was approved by the Chinese Academy of Sciences on December 29,2017 . The approval of the two space missions for solar observations marks a milestone for the Chinese solar physics to glide into the space age.

Since the first artificial satellite was launched by Russia in 1957, about 8000 man-made satellites have been sent to space, among which about 200 satellites were launched by China, including the Beidou navigation satellites [1]. In contrast to NASA and ESA who initiated space missions for astrophysical observations in the 1960s, Chinese astronomers launched their first astronomy-related satellite, Dark Matter Particle Explorer (DAMPE) in December 2015 [2], which was followed by the Hard X-ray Modulation Telescope (HXMT) launched in 2017 [3, 4], and a few more to come [5].

In terms of the solar and space weather observations, the NASA and the ESA have so far launched tens of satellites, the Chinese counterpart, however, contributed little except some small payloads aboard meteorological satellites, communication satellites, or lunar missions $[6,7]$. Therefore, the approval of the two solar space missions in China is a great breakthrough for the thriving Chinese solar physics.

The scientific objectives of the two solar missions are com-

*Corresponding author (email: chenpf@nju.edu.cn) plementary. The ASO-S satellite comprises of three payloads [8], i.e., the Full-disk vector Magnetograph (FMG), the Ly $\alpha$ Solar Telescope (LST) [9], and the Hard X-ray Imager (HXI). They are aimed to measure the magnetic field, to monitor solar flares and coronal mass ejections (CMEs), and to diagnose nonthermal processes during the violent eruptions, respectively. The measurement of the magnetic field is crucial in this mission since the magnetic evolution in the solar atmosphere is the cause for all the eruptions [10]. However, the Solar $\mathrm{H} \alpha$ Imaging Spectrometer telescope will make full scans of the Sun, and obtain the $\mathrm{H} \alpha$ spectral profile of any point throughout the whole solar disk. The scientific objectives of this solar mission are summarized as follows: (1) to detect the slowly-rising filaments in the pre-flare phase [11], which is available only with Doppler measurements for the filaments on the solar disk, not imaging observations; (2) to detect the filament oscillations before eruption, which were proposed to be one possible precursor for CMEs [12]; (3) to detect the plasma dynamics inside post-flare loops and Ellerman bombs [13-15]; (4) to detect Moreton waves associated with big flares and CMEs [16]; (5) to detect the dynamics of solar filaments (or prominences), such as counterstreamings.

It can be seen from the above descriptions that the ASO-S mission is focused on the magnetic field and the solar eruptions, including solar flares and CMEs, whereas the Solar $\mathrm{H} \alpha$ Imaging Spectrometer mission is focused on the precursors for solar eruptions and wave phenomena associated with solar eruptions.

It is worth mentioning that the Solar $\mathrm{H} \alpha$ Imaging Spectrometer mission is a perfect example of collaborations between universities and research institutes. Nanjing University 


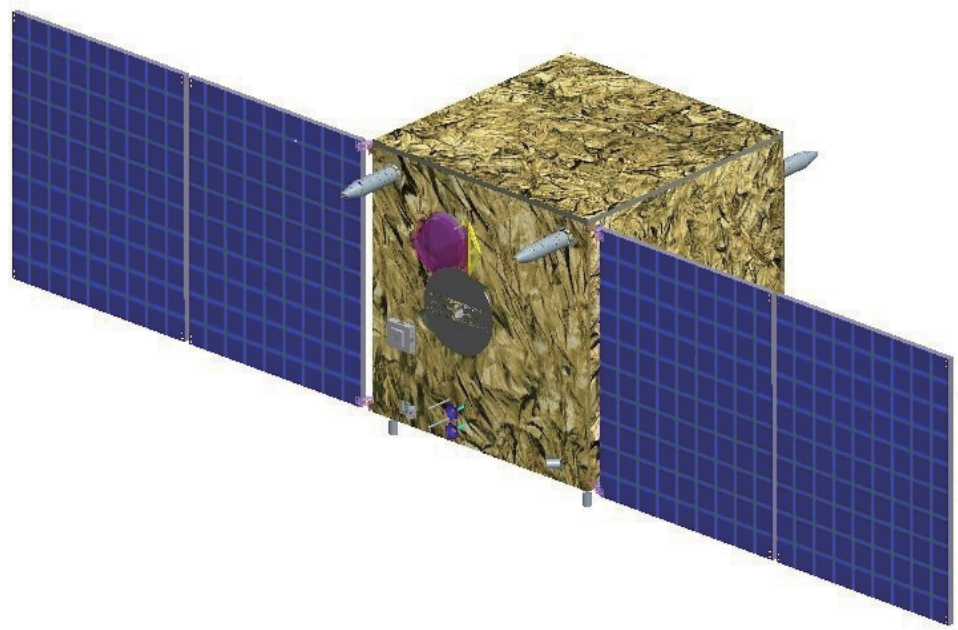

Figure 1 (Color online) Illustration of the proposed Solar $\mathrm{H} \alpha$ Imaging Spectrometer space mission.

is in charge of the scientific definition, data processing, and data management, Changchun Institute of Optics, Fine Mechanics and Physics of the Chinese Academy of Science is responsible for the design and manufacturing of the telescope, whereas the satellite platform is designed by Shanghai Academy of Space Technology.

The Solar $\mathrm{H} \alpha$ Imaging Spectrometer mission, as illustrated in Figure 1, is scheduled to launch in late 2019 or early 2020. Hopefully, solar physicists worldwide will have the chance to analyze the solar observational data obtained by Chinese satellites in the near future.

PFC was financially supported by the Chinese foundations NSFC (11533005) and Jiangsu 333 Project.

1 S. S. Zhou, X. G. Hu, L. Liu, R. Guo, L. F. Zhu, Z. Q. Chang, C. P. Tang, X. Q. Gong, R. Li, and Y. Yu, Sci. China-Phys. Mech. Astron. 59, 109511 (2016).

2 G. Ambrosi, et al. (DAMPE Collaboration), Nature 552, 63 (2017), arXiv: 1711.10981

3 T. P. Li, Nucl. Phys. B-Proc. Suppl. 166, 131 (2007).

4 S. N. Zhang, in China's first dedicated astronomy satellite: The hard $X$-ray modulation telescope (HXMT): American Astronomical Society Meeting Abstracts \#213, volume $\mathbf{4 1}$ of Bulletin of the American Astro- nomical Society (American Astronomical Society, Long Beach, 2009) p. 474.

5 D. Normile, Science 359, 1085 (2018).

6 H. He, C. Shen, H. Wang, X. Zhang, B. Chen, J. Yan, Y. Zou, A. M. Jorgensen, F. He, Y. Yan, X. Zhu, Y. Huang, and R. Xu, Sci. Rep. 6, 32362 (2016).

7 Y. L. Zou, Q. Wang, J. Yan, Y. S. Chen, and Z. Y. Ouyang, Sci. ChinaPhys. Mech. Astron. 60, 049631 (2017).

8 W. Q. Gan, Y. Y. Deng, H. Li, J. Wu, H. Y. Zhang, J. Chang, C. Y. Chen, Z. Q. Zhang, B. Chen, L. Feng, J. H. Guo, Y. M. Hu, Y. Huang, Z. H. Li, Y. M. Peng, D. G. Wang, H. Wang, J. N. Wang, D. S. Wen, Z. Wu, Z. Zhang, and E. X. Zhao, in ASO-S: Advanced Space-based Solar Observatory: Proceedings Volume 9604, Solar Physics and Space Weather Instrumentation VI (SPIE, San Diego, 2015), p. 96040T.

9 H. Li, Proc. IAU 11, 436 (2015).

10 A. R. Choudhuri, Sci. China-Phys. Mech. Astron. 60, 019601 (2017), arXiv: 1612.02544.

11 S. W. Kahler, R. L. Moore, S. R. Kane, and H. Zirin, Astrophys. J. 328, 824 (1988).

12 P. F. Chen, D. E. Innes, and S. K. Solanki, Astron. Astrophys. 484, 487 (2008), arXiv: 0802.1961.

13 Q. M. Zhang, D. Li, and Z. J. Ning, Astrophys. J. 832, 65 (2016), arXiv: 1609.03165 .

14 C. Fang, Q. Hao, M. D. Ding, and Z. Li, Res. Astron. Astrophys. 17, 031 (2017), arXiv: 1702.01905.

15 J. Hong, M. D. Ding, and W. Cao, Astrophys. J. 838, 101 (2017), arXiv: 1703.04268.

16 P. F. Chen, Sci. China-Phys. Mech. Astron. 60, 029631 (2017). 